\title{
Pulmonary Benign Ground-Glass Nodules: CT Features and Pathological Findings
}

This article was published in the following Dove Press journal:

International Journal of General Medicine

\section{Wang-Jia Li ${ }^{1}{ }^{1} *$ \\ Fa-Jin Lv'** \\ Yi-Wen Tan ${ }^{2}$ \\ Bin-Jie Fu (D)' \\ Zhi-Gang Chu (D)'}

'Department of Radiology, The First Affiliated Hospital of Chongqing Medical University, Chongqing, People's Republic of China; ${ }^{2}$ Department of Pathology, The First Affiliated Hospital of Chongqing Medical University, Chongqing, People's Republic of China

*These authors contributed equally to this work
Correspondence: Zhi-Gang Chu Department of Radiology, The First Affiliated Hospital of Chongqing Medical University, I\# Youyi Road, Yuanjiagang, Yuzhong District, Chongqing, 400016,

People's Republic of China

Tel +8618723032809

Fax +8623688II487

Email chuzg0815@I63.com
Background: Some pulmonary ground-glass nodules (GGNs) are benign and frequently misdiagnosed due to lack of understanding of their CT characteristics. This study aimed to reveal the CT features and corresponding pathological findings of pulmonary benign GGNs to help improve diagnostic accuracy.

Patients and Methods: From March 2016 to October 2019, patients with benign GGNs confirmed by operation or follow-up were enrolled retrospectively. According to overall CT manifestations, GGNs were classified into three types: I, GGO with internal high-attenuation zone; II, nodules lying on adjacent blood vessels; and other type, lesions without obvious common characteristics. CT features and pathological findings of each nodule type were evaluated.

Results: Among the 40 type I, 25 type II, and 14 other type GGNs, 24 (60.0\%), 19 (76.0\%), and $10(71.4 \%)$ nodules were resected, respectively. Type I GGNs were usually irregular (25 of $40,62.5 \%$ ) with only one high-attenuation zone (38 of $40,95.0 \%$ ) (main pathological components: thickened alveolar walls with inflammatory cells, fibrous tissue, and exudation), which was usually centric ( 24 of $40,60.0 \%$ ), having blurred margin ( 38 of $40,95.0 \%$ ), and connecting to blood vessels ( 32 of $40,80.0 \%$ ). The peripheral GGO (main pathological component: a small amount of inflammatory cell infiltration with fibrous tissue proliferation) was usually ill-defined (28 of 40, 70.0\%). Type II GGNs (main pathological components: focal interstitial fibrosis with or without inflammatory cell infiltration) lying on adjacent vessel branches were usually irregular (19 of $25,76.0 \%)$ and well defined (16 of $25,64.0 \%)$ but showed coarse margins (15 of 16, 93.8\%). Other type GGNs had various CT manifestations but their pathological findings were similar to that of type II.

Conclusion: For subsolid nodules with CT features manifested in type I or II GGNs, follow-up should be firstly considered in further management.

Keywords: ground-glass nodule, benignity, CT, pathology, lung

\section{Introduction}

The advent of CT screening for lung cancer has increased the incidence of groundglass nodules (GGNs). GGNs, especially part-solid GGNs, are more likely to be lung cancer than solid nodules. ${ }^{1-4}$ Furthermore, most part-solid GGNs are confirmed as minimally invasive adenocarcinomas or invasive adenocarcinomas, often requiring surgical resection. ${ }^{5,6}$ Although GGNs have a high probability of malignancy, quite a few lesions were benign. Among them, most are transient and a small number of persistent lesions are finally confirmed as focal interstitial fibrosis. ${ }^{7-9}$ These GGNs are often misdiagnosed as lung cancers and treated by unnecessary surgical resection. Therefore, carefully discriminating benign GGNs from malignant ones remains a challenge. 
Previous studies have investigated the differences in CT features between benign and malignant GGNs and found that larger size, lobulation, spiculation, air bronchogram, and solid component were indicative of malignancy. ${ }^{10-13}$ However, Felix et $\mathrm{al}^{14}$ found that the resolving GGNs were more likely to be lobular, polygonal, mixed, and larger than persistent GGNs, which was contrary to the results of other studies. Additionally, these studies only revealed the relative CT features of benign GGNs but failed to summarize their common CT characteristics, resulting in limited differential diagnostic value. Therefore, further studies should focus on determining whether benign GGNs have some distinct features to help improve the diagnostic accuracy of benign GGNs.

In clinical practice, we found that benign GGNs had various CT manifestations, but some of them had similar CT features, which were not well studied. We hypothesize that benign GGNs have some relatively consistent CT characteristics, which might be related to pathological findings. Therefore, this study aimed to reveal the CT features and corresponding pathological findings of pulmonary benign GGNs to help improve diagnostic accuracy.

\section{Patients and Methods}

This retrospective study used non-identifiable data and have no potential risks to patients, so the study was approved by ethics committee of The First Affiliated Hospital of Chongqing Medical University and the requirement for informed consent was waived. This study was conducted in accordance with the Declaration of Helsinki.

\section{Patients}

From March 2016 to October 2019, patients with surgically resected and pathologically confirmed benign GGNs or GGNs resolved during follow-up were enrolled retrospectively. Inclusion criteria were as follows: (1) the lesion was a GGN (diameter, $\leq 3 \mathrm{~cm}$ ); (2) availability of thinsection CT images with a thickness $\leq 1 \mathrm{~mm}$; (3) the patients' clinical and pathological data were complete for those with resected GGNs. Exclusion criteria were as follows: (1) patients with diffused lesions, which would influence the evaluation; and (2) CT images contained significant motion artifacts. Finally, 79 patients with benign GGNs were enrolled in this study. The patient selection procedure is shown in Figure 1.

\section{CT Examinations}

All patients were scanned on a 128 -slice MDCT scanner (Somatom Definition FLASH, Siemens Healthcare, Forchheim, Germany) at the end of inspiration during a single breath-hold. Non-contrast CT examinations were performed with the following parameters: tube voltage, $110-120 \mathrm{kV}$; tube current, $50-150 \mathrm{mAs}$; beam pitch, 1.0; detector collimation, $0.6 \mathrm{~mm}$; rotation time, $0.5 \mathrm{~s}$. All patients were scanned from the thoracic inlet to the lung base. The data were reconstructed into the images with slice thickness $\leq 1 \mathrm{~mm}$ using a high-resolution reconstruction kernel and displayed with a standard lung window setting (width, $1600 \mathrm{HU}$; level, $-600 \mathrm{HU}$ ).

\section{CT Data Analysis}

All patients' CT data were independently reviewed on a PACS workstation (Carestream Vue PACS) by 2 radiologists (Z.G.C. and F.J.L.) with more than 10 years' experience of chest CT interpretation, who were blinded to the pathological results of nodules. Any divergences of the two radiologists during evaluation were resolved by consensus. Data analysis was performed on both the original and reformatted images.

The overall manifestations of benign GGNs were evaluated, and most of them were manifested as ground-glass opacities (GGOs) with internal high-attenuation zone and GGNs lying on adjacent blood vessels. Therefore, based on the overall CT manifestations, GGNs were classified into three types: type I: ground-glass opacity $(\mathrm{GGO})$ with internal high-attenuation zone (CT value of highattenuation zone is at least $200 \mathrm{HU}$ higher than GGO) (Figure 2A); Type II: GGN lying on adjacent blood vessels (Figure 2B); Other type: GGNs with other various CT features rather than those manifested in type I and II nodules. Type II and other type GGNs had no significant high-attenuation zone. The following CT features were further evaluated for each GGN type: nodule location, shape (round/oval or irregular), diameter (mean of the longest diameter and perpendicular diameter on axial images), border (well defined or ill defined), and margin of well defined lesion (smooth or coarse). Additionally, for type I GGNs, the number, location, shape, diameter, and edge (clear or blurred) of high-attenuation zone, whether the high-attenuation zone connected to blood vessels (artery or vein), and the uniformity of peripheral GGO component (homogeneous or heterogeneous) were assessed. For type II GGNs, the uniformity of nodules 
Patients with pulmonary ground-glass nodule (GGN) on thin-section CT images between March 2016 and July 2019 (n=1467)

\section{Exclusion:}

GGNs pathologically confirmed as malignancy $(\mathrm{n}=1135)$ persistent GGNs without operation $(\mathrm{n}=181)$

Patients with diffused lesions distributed around GGNs (n=72)

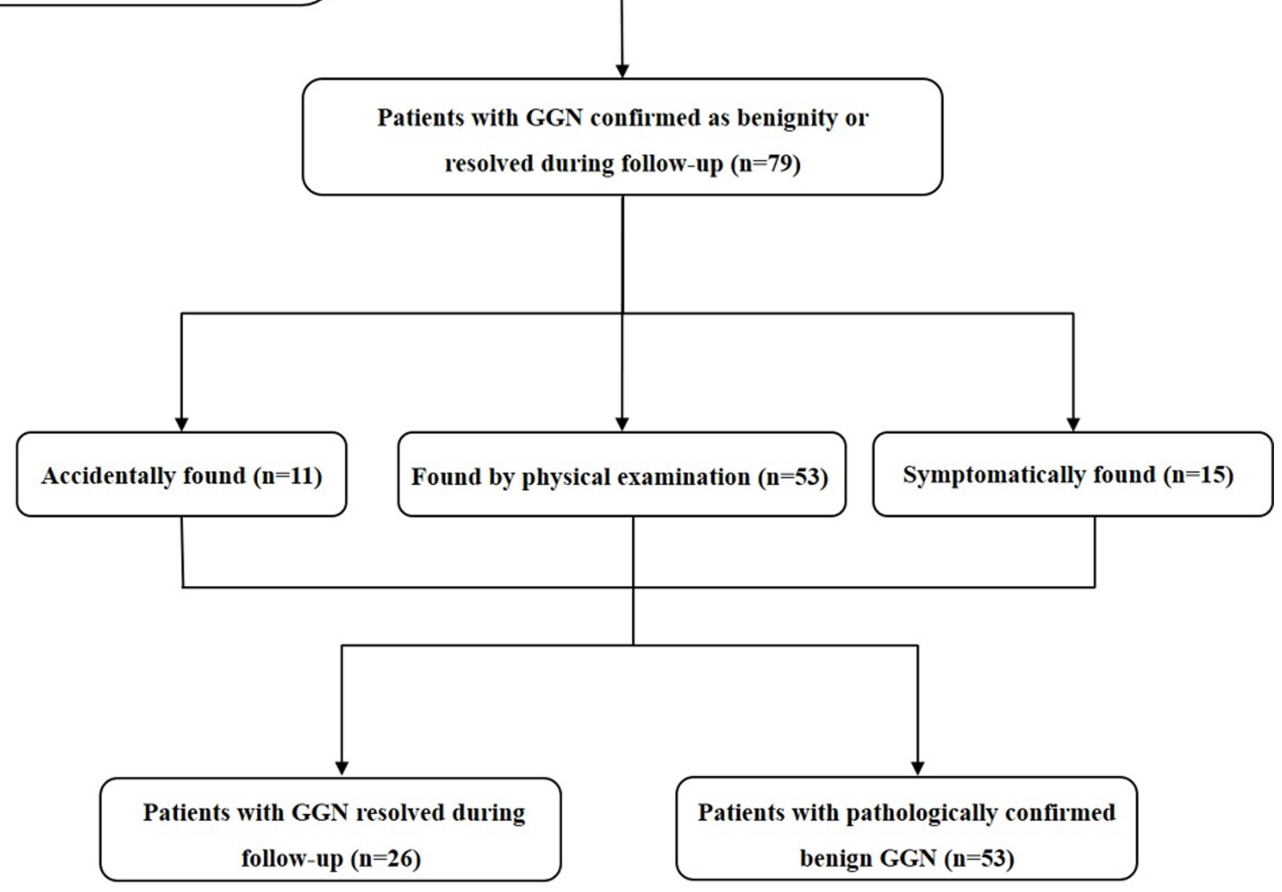

Figure I Flowchart of study population.

Abbreviation: GGN, ground-glass nodule.

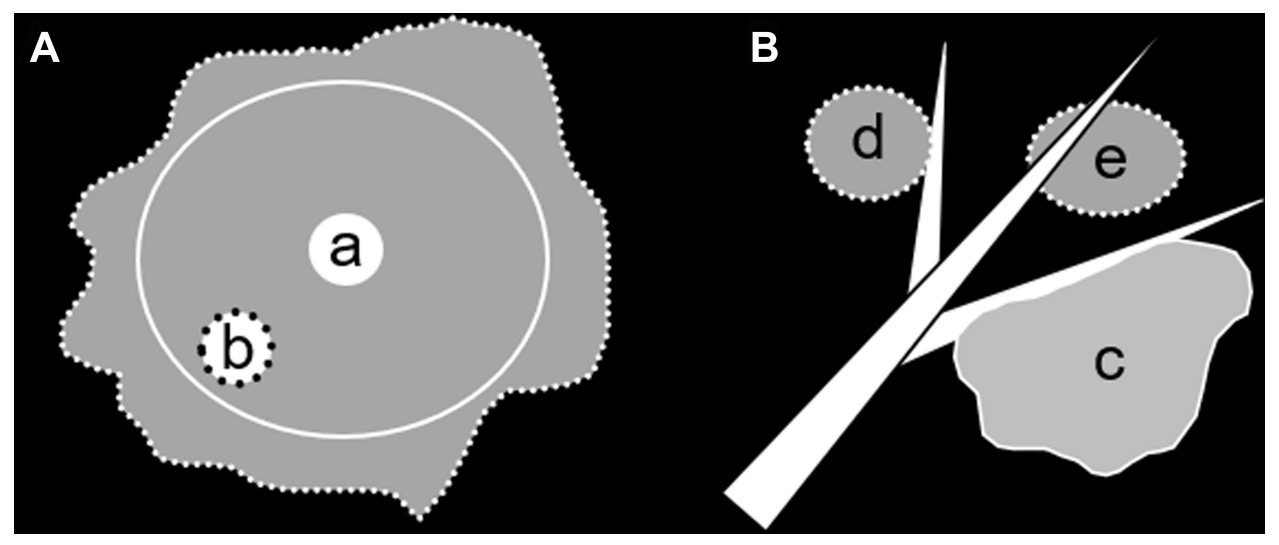

Figure 2 CT manifestations of type I and II GGNs. (A) Type I GGN is defined as a regular (marked by white solid line) or irregular (marked by white dotted line) GGO containing an internal centric (a) or eccentric (b) high-attenuation zone. (B) Type II GGN is defined when one nodule edge abuts an adjacent blood vessel, and the length of this edge close to the vessel is more than or equal to two-thirds of the nodule's long diameter on the same section (c). The GGNs locally cling to the blood vessels (d) or surrounding the blood vessels (e) could not be seen as type II lesions.

Abbreviations: GGN, ground-glass nodule; GGO, ground-glass opacity. 
Table I Clinical Features of Patients with Different Types of Benign GGNs

\begin{tabular}{|c|c|c|c|c|}
\hline Clinical Features & Type I $(n=40)$ & Type II $(n=25)$ & Other Type $(n=14)$ & $P$ value \\
\hline \multicolumn{5}{|l|}{ Sex } \\
\hline Man & $20(50.0)$ & $5(20.0)$ & $7(50.0)$ & $0.041^{\ddagger}$ \\
\hline Woman & $20(50.0)$ & $20(80.0)$ & $7(50.0)$ & \\
\hline Age (y) & $53 \pm 11$ & $54 \pm 11$ & $50 \pm 10$ & $0.838^{\&}$ \\
\hline Current or former smoker & $18(45.0)$ & $2(8.0)$ & $6(42.9)$ & $0.003^{\S}$ \\
\hline Current or former drinker & $16(40.0)$ & I (4.0) & $3(21.4)$ & $0.003^{\S}$ \\
\hline Surgically resected & $24(60.0)$ & $19(76.0)$ & $10(71.4)$ & - \\
\hline Resolved in follow-up & $16(40.0)$ & $6(24.0)$ & $4(28.6)$ & - \\
\hline Follow-up time (month)* & $4.5 \pm 4.4$ & $2.4 \pm 0.5$ & $7.4 \pm 5.4$ & - \\
\hline \multicolumn{5}{|l|}{ Mode of detections } \\
\hline Accidentally found & $4(10.0)$ & $4(16.0)$ & $3(21.4)$ & \\
\hline Found by physical examination & $29(72.5)$ & $14(56.0)$ & $10(71.4)$ & $0.413^{\S}$ \\
\hline Symptomatically found & $7(17.5)$ & $7(28.0)$ & I (7.I) & \\
\hline Malignancy history & $2(5.0)$ & I (4.0) & I (7.I) & - \\
\hline WBC $\left(\mathrm{uL}^{-1}\right)$ & $6872 \pm 2931$ & $6131 \pm 2900$ & $6087 \pm 1733$ & $0.296^{8}$ \\
\hline Eosinophil (uL $\left.\mathrm{L}^{-1}\right)$ & $154 \pm 223$ & $94 \pm 100$ & $70 \pm 38$ & $<0.001^{8}$ \\
\hline
\end{tabular}

Notes: Data are presented as $\mathrm{n}(\%)$ or Means \pm standard deviation. *Data are collected from patients with resolved GGNs. ${ }^{\ddagger}$ Calculated with the Pearson $\chi 2$ test. ${ }^{8}$ Calculated with the Fisher's exact test. ${ }^{8}$ Wilcoxon rank sum test.

Abbreviations: GGN, ground-glass nodule; WBC, white blood cell.

Table 2 Thin-Section CT Features of Different Types of Benign GGNs

\begin{tabular}{|c|c|c|c|c|}
\hline CT Features & Type I $(n=40)$ & Type II (n = 25) & Other Types $(n=14)$ & $P$ value \\
\hline \multicolumn{5}{|l|}{ Lesion location } \\
\hline Right upper lobe & $15(37.5)$ & $13(52.0)$ & $6(42.9)$ & $0.384^{\S}$ \\
\hline Right middle lobe & $2(5.0)$ & $2(8.0)$ & $4(28.6)$ & \\
\hline Right lower lobe & $7(17.5)$ & $4(16.0)$ & I (7.I) & \\
\hline Left upper lobe & II (27.5) & $4(16.0)$ & $3(21.4)$ & \\
\hline Left lower lobe & $5(12.5)$ & $2(8.0)$ & $0(0.0)$ & \\
\hline Mean diameter $(\mathrm{mm})$ & $11.4 \pm 4.8$ & $9.2 \pm 3.3$ & $9.8 \pm 2.9$ & $0.299^{\&}$ \\
\hline \multicolumn{5}{|c|}{ Uniformity of GGO or GGN } \\
\hline Homogeneous & $18(45.0)$ & $12(48.0)$ & $4(28.6)$ & - \\
\hline Heterogenous & $22(55.0)$ & $13(52.0)$ & $10(71.4)$ & \\
\hline \multicolumn{5}{|l|}{ Lesion shape } \\
\hline Round/oval & $15(37.5)$ & $6(24.0)$ & $4(28.6)$ & $0.527^{\S}$ \\
\hline Irregular & $25(62.5)$ & $19(76.0)$ & $10(7 \mid .4)$ & \\
\hline \multicolumn{5}{|l|}{ Lesion border } \\
\hline III defined & $28(70.0)$ & $9(36.0)$ & $5(35.7)$ & $0.010^{\ddagger}$ \\
\hline well defined & $12(30.0)$ & $16(64.0)$ & $9(64.3)$ & \\
\hline \multicolumn{5}{|c|}{ Margin of well definednodules } \\
\hline Coarse & II (9I.7) & $15(93.8)$ & $7(77.8)$ & $0.540^{\S}$ \\
\hline Smooth & $\mathrm{I}(8.3)$ & $\mathrm{I}(6.3)$ & $2(22.2)$ & \\
\hline
\end{tabular}

Notes: Data are presented as $n(\%)$ or Means \pm standard deviation. ${ }^{\ddagger}$ Calculated with the Pearson $\chi 2$ test. ${ }^{8}$ Calculated with the Fisher's exact test. ${ }^{8}$ Wilcoxon rank sum test. Abbreviations: GGN, ground-glass nodule; GGO, ground-glass opacity. 
and the adjacent blood vessels (artery or vein; normal, dilated, or distorted) lied by nodules were evaluated. When the interpretations of two radiologists differed, the divergences were resolved by consensus.

\section{Statistical Analysis}

All statistical analyses were performed using the Statistical Package for Social Sciences program (version 22.0, IBM, Armonk, NY). Continuous data are presented as mean \pm standard deviation, while categorical data are presented as numbers and percentages. Statistical differences of three GGN types were analyzed using the Wilcoxon rank sum test for patient age, WBC and eosinophil count, and mean diameter of GGN. And patient's sex, smoking and drinking history, mode of detection, and CT characteristics (lesion location, shape, border, and margin of well defined nodules) were analyzed using the Pearson $\chi^{2}$ test and Fisher exact test, as appropriate. A $\mathrm{P}$ value $<0.05$ indicated a significant difference.

\section{Results}

\section{Patients' Clinical Characteristics}

Seventy-nine patients (mean age: $53 \pm 11$ years; range: 19-79 years) were included, comprising 32 (40.5\%) men (mean age: $55 \pm 10.5$ years; range: 36-79 years) and $47(59.5 \%)$ women (mean age: $51 \pm 11$ years; range: 19-68 years). Among 79 patients with GGNs, $53(67.1 \%)$ were confirmed by surgical resection and pathological examination, and $26(32.9 \%)$ by disappearance during follow-up (mean follow-up time: 4.8 months \pm 4.5 ; range: $0.5 \sim 16$ months). A total of 24 resected GGNs had follow-up results (mean follow-up time: 3.0 months \pm 2.9 ; range: $0.3 \sim 8.5$ months), 22 (91.7\%) GGNs had no significant change, and 2 (8.3\%) GGNs showed a slight decrease in size or density. At 3 months after first detection, most of transient GGNs $(19 / 26,73.1 \%)$ resolved, whereas most of resected nodules $(22 / 24,91.7 \%)$ remained stable.

Among 79 patients, there were 40 (50.6\%), 25 $(31.6 \%)$, and $14(17.7 \%)$ patients with type I, type II,

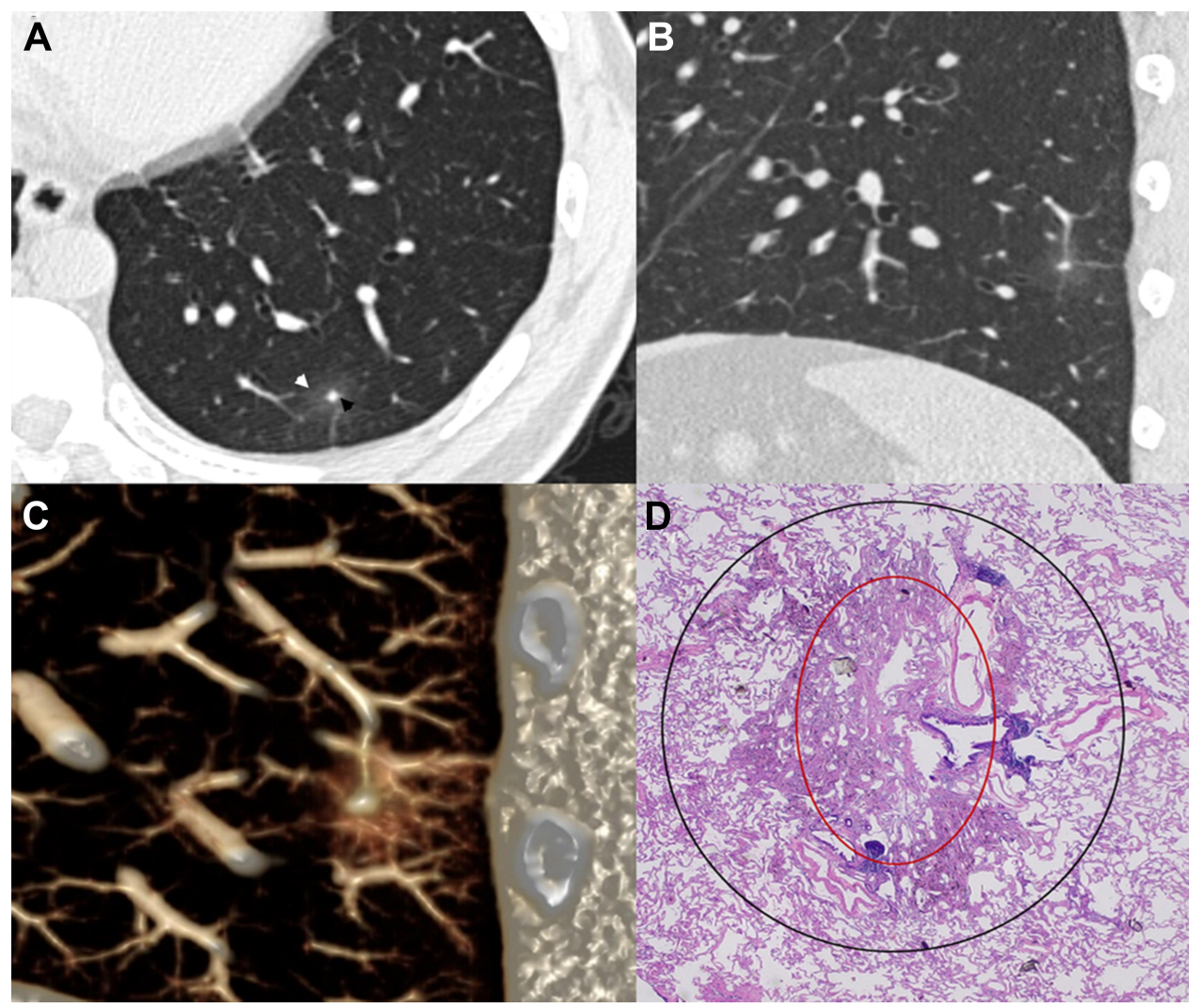

Figure $3 \mathrm{~A}$ patient with a type I GGN confirmed by surgical operation. Axial CT image (A) shows a 16.6 mm round mixed GGN located in the left lower lobe, the internal high-attenuation zone (black arrow) is centric, round, and blurred, and the peripheral GGO (white arrow) is ill-defined. Sagittal CT image (B) and VR image (C) confirm that the internal high-attenuation zone is connected to an adjacent blood vessel. Histopathologic analysis (D) reveals thickened alveolar walls with inflammatory cells, fibrous tissue and exudation in high-attenuation (red circle) zone and a small amount of inflammatory cell infiltration in the peripheral GGO zone (black circle).

Abbreviations: GGN, ground-glass nodule; GGO, ground-glass opacity. 


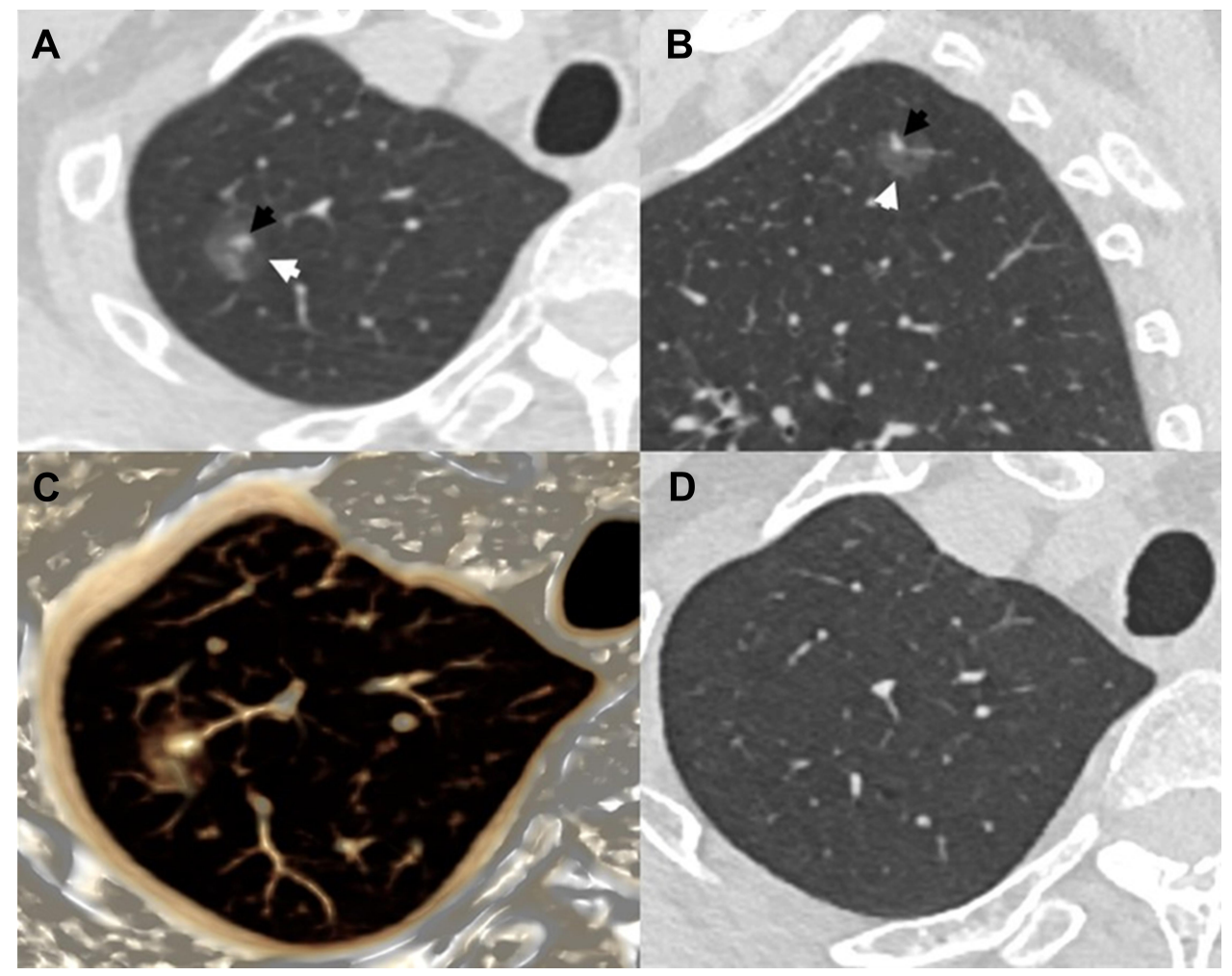

Figure 4 A patient with a transient type I GGN. He had blood eosinophilia (eosinophil count, 620/ul). Axial (A) and sagittal (B) CT images show a I3.7 mm oval mixed GGN located in the right upper lobe, the internal high-attenuation (black arrow) zone is eccentric, irregular, and blurred, and the peripheral GGO (white arrow) is partly ill-defined. VR image (C) confirm that the internal high-attenuation zone is connected to an adjacent blood vessel. At follow-up CT (D) obtained 2.5 months later, the GGN has disappeared.

Abbreviations: GGN, ground-glass nodule; GGO, ground-glass opacity.

and other type GGNs, respectively. There were more women $(\mathrm{P}=0.041)$, nonsmokers $(\mathrm{P}=0.003)$ and nondrinkers $(\mathrm{P}=0.003)$ in type II than in other two types. Eosinophil count was higher in patients with type I GGNs than that in patients with type II and other type lesions $(\mathrm{P}<0.001)$, and eosinophilia was observed in 3 patients with type I GGNs. Patients' clinical characteristics are summarized in Table 1.

\section{CT Features of Different Types of Benign GGNs}

CT features of each GGN type are summarized in Table 2. There were no significant differences in their location, diameter, shape, and margin of well defined nodules among different types $(\mathrm{P}=0.384, \mathrm{p}=0.299$, $\mathrm{P}=0.527$, and $\mathrm{P}=0.540$, respectively), whereas there was a significant difference in lesion border $(\mathrm{P}=$ 0.010). Benign GGNs were mainly located in the upper lobes and had an irregular shape. Type II and other type GGNs were usually well defined but had coarse margins. For each type of benign GGNs, there were no significant differences in their CT features between resolving and resected ones, except for the diameter in type II $(\mathrm{P}=0.006)$.

For type I GGNs, the peripheral GGO components were frequently ill-defined $(28 / 40,70.0 \%)$. Most nodules $(38 / 40,95.0 \%)$ only had a single highattenuation zone with a mean diameter of $3.5 \mathrm{~mm}$ (range: $1.2 \sim 7.5 \mathrm{~mm}$ ). Among the 40 highattenuation zones, $24(60.0 \%)$ were centric and 16 $(40.0 \%)$ were eccentric, $22(55.0 \%)$ were regular (round or oval) and $18(45.0 \%)$ were irregular, 38 $(95.0 \%)$ had blurred edge and 2 (5.0\%) had clear edge, and $32(80.0 \%)$ connected to adjacent blood vessels (arteries: 20/32, 62.5\%, veins: 12/32, 37.5\%) (Figures 3 and 4).

For 25 type II GGNs, 14 (56.0\%) abutted the pulmonary artery branches and $11(44.0 \%)$ abutted the pulmonary vein branches, but these vessels had no significant changes in morphology (Figures 5 and 6). In addition, 12 (48.0\%) and $13(52.0 \%)$ cases were homogeneous and heterogeneous, respectively. 

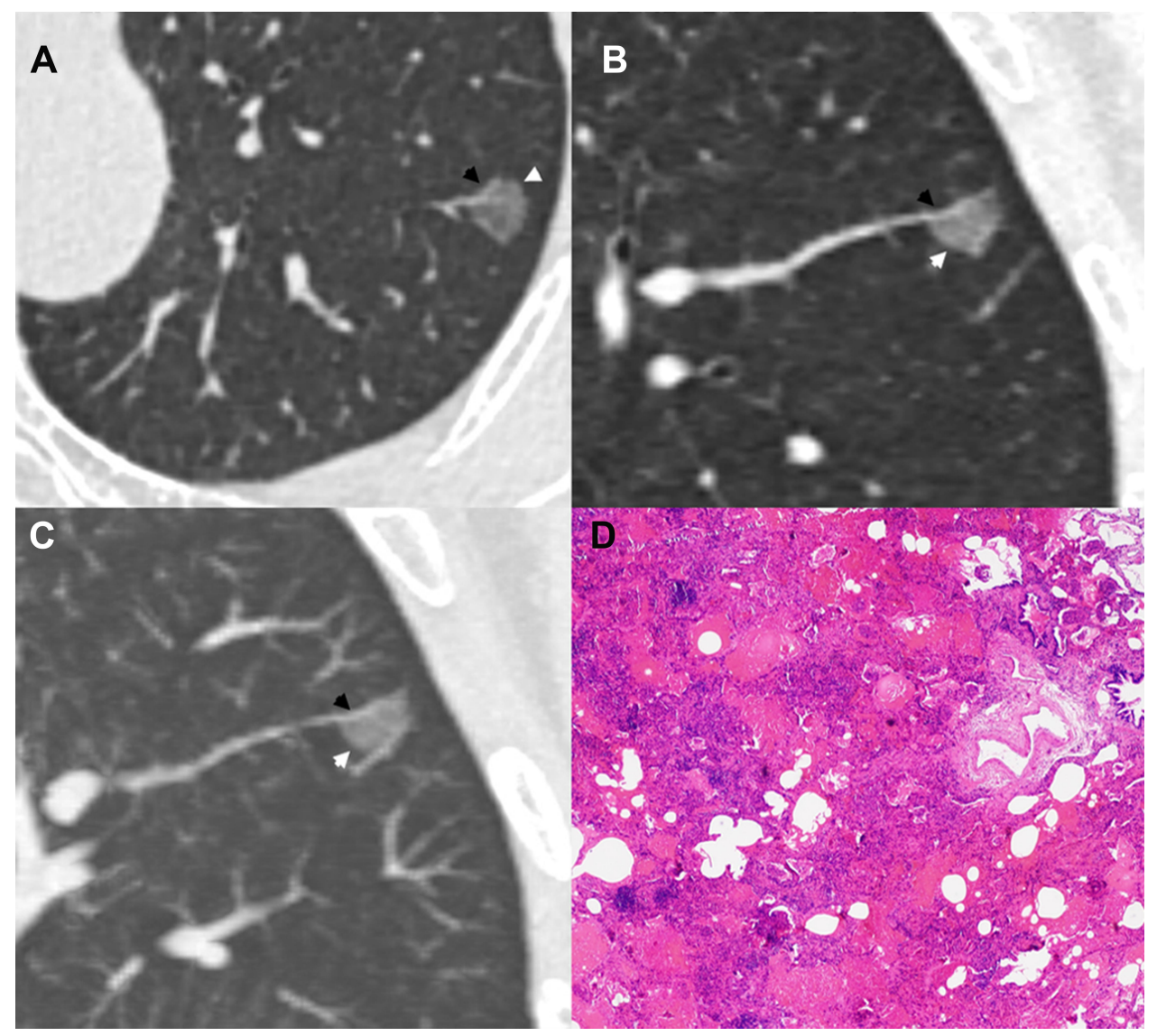

Figure 5 A patient with a type II GGN confirmed by surgical operation. Axial CT image (A) shows a 9 mm round well defined GGN (white arrow) with slightly coarse margin located in the left upper lobe. A blood vessel (black arrow) is close to the edge of this lesion. MPR (B) and MIP (C) images confirm that one edge of this nodule (white arrow) widely abuts the upper blood vessel (black arrow). Histopathologic analysis (D) reveals focal interstitial fibrosis with inflammatory cell infiltration.

Abbreviations: GGN, ground-glass nodule; MPR, multiplanar reconstruction; MIP, maximum intensity projection.

Fourteen other type GGNs included 5 (35.7\%) lesions with bubble lucency, $4(28.6 \%)$ clinging to pleura or fissure with a wide base, $3(21.4 \%)$ with faint density and ill-defined border, and $2(14.3 \%)$ with a significant irregular shape.

\section{Pathological Findings}

The pathological findings of 53 surgically resected GGNs were reviewed. In 24 type I GGNs, the pathological components were thickened alveolar walls with inflammatory cells, fibrous tissue and exudation (22, 91.7\%) and granuloma (2, $8.3 \%$ ) for internal high-attenuation zones, and a small amount of inflammatory cell infiltration with $(21,87.5 \%)$ and without $(3,12.5 \%)$ fibrous tissue proliferation for peripheral GGOs. The main pathological findings were similar for 19 type II and 10 other type GGNs, including focal interstitial fibrosis (type II GGNs: $8 / 19,42.1 \%$; other type GGNs: $4 / 10,40.0 \%$ ) and fibrous tissue proliferation with inflammatory cell infiltration (type II GGNs: 11/19, 57.9\%; other type GGNs: 6/10, 60.0\%).

\section{Discussion}

Pulmonary GGNs have a high probability of being malignant, but some of them are benign, frequently misdiagnosed as lung cancers and treated by unnecessary surgical resection. Thus, being able to recognize benign GGNs by $\mathrm{CT}$ is important. Previous studies failed to summarize the common features predictive of benign GGNs. ${ }^{15,16}$ In this study, we investigated benign GGNs and found that they had some common CT features, which could be explained well by their pathological findings. These new findings may be helpful for distinguishing the potential benign GGNs.

Among benign GGNs, type I lesions were the most common. Their internal high-attenuation zones were mostly single, usually having blurred edge and connecting to the adjacent blood vessels. And the peripheral GGOs were usually ill-defined, which was consistent with a previous study. ${ }^{17}$ Pathologically, the high-attenuation zone within GGN represents the inflammation and 


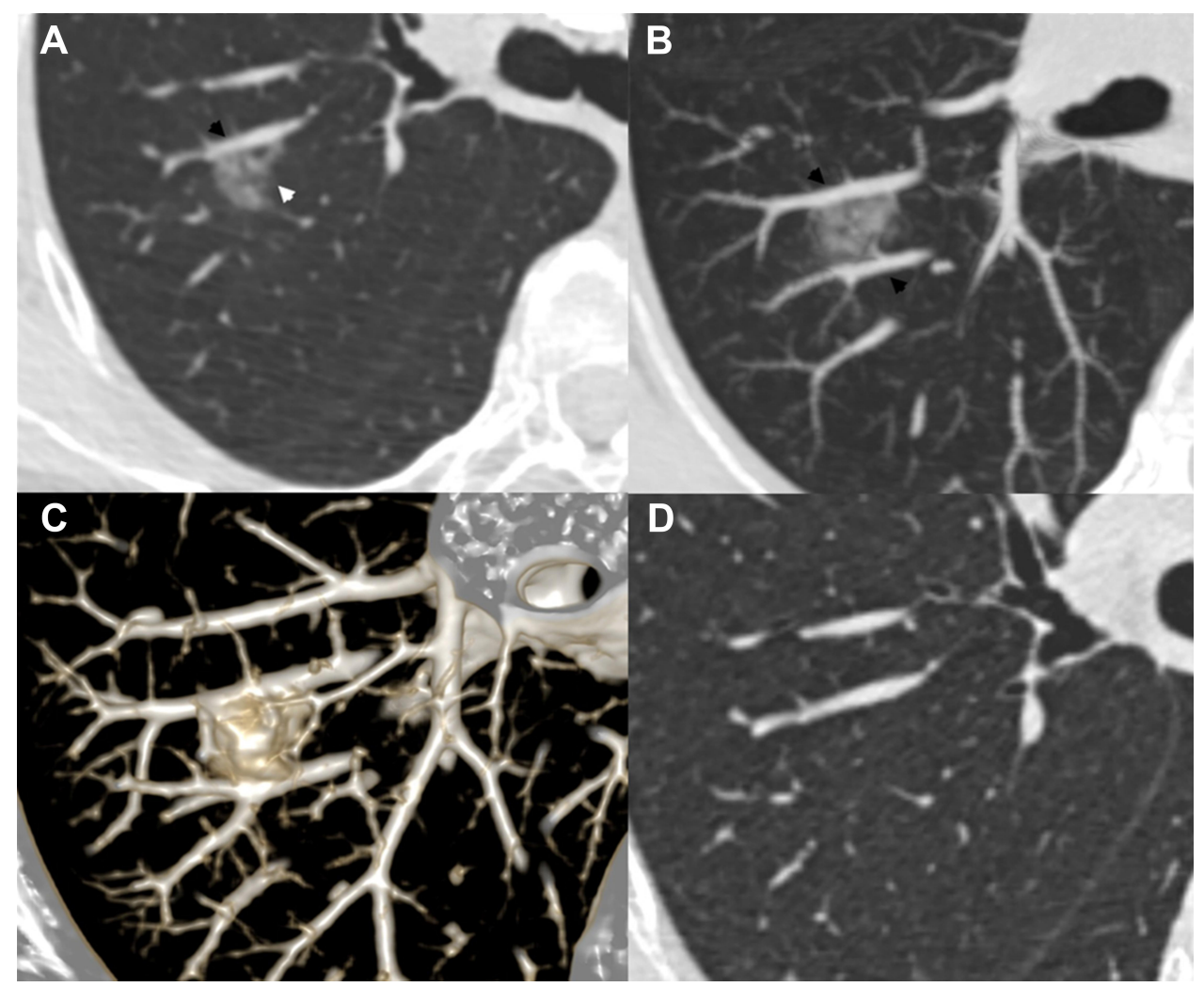

Figure 6 A patient with a transient type II GGN. Axial CT image (A) shows a $15.6 \mathrm{~mm}$ well defined GGN (white arrow) with slightly coarse margin located in the right upper lobe. One blood vessel (black arrow) is close to the edge of this lesion. MIP (B) and VR (C) images confirm that two edges of this nodule abut adjacent blood vessels (black arrows). Follow-up CT scan (D) performed 3 months later shows the disappearance of this lesion.

Abbreviations: GGN, ground-glass nodule; VR, volume rendering reconstruction; MIP, maximum intensity projection.

exudation, which lead to its blurred edge. In addition, inflammations in high-attenuation zone were more severe than those in the peripheral GGO area. This indicates that the high-attenuation zone may be the primary lesion originating from terminal bronchioles, while the peripheral GGO was just caused by it.

Oh et $\mathrm{al}^{18}$ reported that transient GGN, especially transient part-solid GGN, might result from eosinophil infiltration and blood eosinophil count was expected to be an indicator of lesion benignity or malignancy. However, blood eosinophilia showed very low sensitivity. ${ }^{19}$ In this study, only 3 patients with type I GGNs had eosinophilia. Thus, pulmonary eosinophil infiltration might be responsible for a very small portion of benign part-solid GGNs.

The most noteworthy feature of type II GGNs was that lesions abutted adjacent blood vessels but did not surround and affect them. This manifestation may be related to the peripheral interstitium of blood vessels, which possibly act as a barrier to inflammatory extension. ${ }^{20}$ To determine the relationship between nodules and blood vessels, multiplanar reconstruction (MPR) is a basic tool. When a blood vessel is close to the edge of a GGN or a GGN locates at a vessel bifurcation on axial images, MPR is essential for evaluating the relationship between lesions and blood vessels.

It is worth mentioning that part-solid GGNs are also frequently detected in malignancy. However, in our experience, and those from the literature, solid components in malignant GGNs are often irregular and have coarse margins or present as multiple spots, ${ }^{21}$ and the peripheral GGOs are usually well defined. In contrast to type II benign GGNs, malignant GGNs tended to wrap and drag vessels into lesions. ${ }^{22}$ Gao et $\mathrm{al}^{22}$ and $\mathrm{Lv}$ et $\mathrm{al}^{23}$ found that the blood vessels passing through the nodules without any changes were commonly observed in both benign and malignant GGNs, but distorted and/or dilated vessels were more frequently observed in malignant GGNs. These differences could help to differentiate benign and malignant GGNs.

Most benign GGNs could resolve during follow-up, but a few persist. The guidelines of British Thoracic Society and the Fleischner Society recommend an initial follow-up 
CT scan 3 months after the first detection, ${ }^{24,25}$ which is an appropriate interval to wait before determining the possible intervention. ${ }^{26,27}$ In this study, the mean resolved time of transient GGNs was 4.8 months and most GGNs disappeared within 3 months, which was consistent with a previous report. ${ }^{18}$ However, most of the resected nodules with follow-up CT remained stable at 3 months. This difference may be related to different pathological components. Therefore, although some nodules with these certain features had no change at 3 months, follow-up was still recommended.

There are several limitations in this study. First, the benign GGNs were confirmed by operation or follow-up. Although GGNs were confirmed as benignity through different ways, this brings us more knowledge. For the nodules with similar manifestations, some could provide information about their pathological components and others indicate whether they would resolve in the future. Second, it was unknown whether CT features presented in type I and II GGNs could be found in malignant GGNs. This study primarily aimed to find those nodules requiring follow-up after the first detection by identifying the common CT features of benign GGNs, but not to determine their unique characteristics. Our next study will be comparing the benign and malignant GGNs with similar CT features, such as the part-solid nodules. Third, a few patients with benign GGNs in this study still had no definite features because of small sample sizes. Further studies with larger sample sizes are required.

\section{Conclusion}

In conclusion, benign GGNs have some common CT features. Nodules with isolated, blurred internal high-attenuation zones connecting to the blood vessels and peripheral ill-defined GGO (Type I), or nodules abutting but not surrounding blood vessels (Type II) are likely to be benign and might be resolved during follow-up. Therefore, when GGNs with these certain characteristics are detected, follow-up should be firstly considered in view of their higher proportion among benign GGNs, which may be helpful to reduce the unnecessary operations of non-cancerous GGNs.

\section{Acknowledgments}

This work was supported by the National Natural Science Foundation of China (81601545) and Chongqing Health and Family Planning Commission Foundation (2016MSXM018) of China.

\section{Disclosure}

The authors report no conflicts of interest in this work.

\section{References}

1. Mao R, She Y, Zhu E, et al. Proposal for restaging of invasive lung adenocarcinoma manifesting as pure ground glass opacity. Ann Thorac Surg. 2019;107:1523-1531. doi:10.1016/j.athoracsur.20 18.11.039

2. She Y, Zhao L, Dai C, et al. Preoperative nomogram for identifying invasive pulmonary adenocarcinoma in patients with pure ground-glass nodule: a multi-institutional study. Oncotarget. 2017;8:17229-17238. doi:10.18632/oncotarget.11236

3. Henschke CI, Yankelevitz DF, Mirtcheva R, et al. CT screening for lung cancer: frequency and significance of part-solid and nonsolid nodules. Am J Roentgenol. 2002;178:1053-1057. doi:10.2214/ ajr.178.5.1781053

4. Kim HY, Shim YM, Lee KS, Han J, Yi CA, Kim YK. Persistent pulmonary nodular ground-glass opacity at thin-section CT: histopathologic comparisons. Radiology. 2007;245:267-275. doi:10.1148/ radiol.2451061682

5. Kim H, Park CM, Koh JM, Lee SM, Goo JM. Pulmonary subsolid nodules: what radiologists need to know about the imaging features and management strategy. Diagn Interv Radiol. 2014;20:47-57. doi:10.5152/dir.2013.13223

6. Tsutani Y, Miyata Y, Yamanaka T, et al. Solid tumors versus mixed tumors with a ground-glass opacity component in patients with clinical stage IA lung adenocarcinoma: prognostic comparison using high-resolution computed tomography findings. $J$ Thorac Cardiovasc Surg. 2013;146:17-23. doi:10.1016/j.jtcvs.2012.11.019

7. Park CM, Goo JM, Lee HJ, Lee CH, Chun EJ, Im JG. Nodular ground-glass opacity at thin-section CT: histologic correlation and evaluation of change at follow-up. Radiographics. 2007;27:391-408. doi: $10.1148 /$ rg. 272065061

8. Park CM, Goo JM, Lee HJ, et al. Focal interstitial fibrosis manifesting as nodular ground-glass opacity: thin-section CT findings. Eur Radiol. 2007;17:2325-2331. doi:10.1007/s00330-007-0596-z

9. Austin JH, Garg K, Aberle D, et al. Radiologic implications of the 2011 classification of adenocarcinoma of the lung. Radiology. 2013;266:62-71. doi:10.1148/radiol.12120240

10. Oda S, Awai K, Liu D, et al. Ground-glass opacities on thin-section helical CT: differentiation between bronchioloalveolar carcinoma and atypical adenomatous hyperplasia. AJR Am $J$ Roentgenol. 2008;190:1363-1368. doi:10.2214/AJR.07.3101

11. Lee HJ, Goo JM, Lee CH, et al. Predictive CT findings of malignancy in ground glass nodules on thin-section chest CT: the effects on radiologist performance. Eur Radiol. 2009;19:552-560. doi:10.1007/s00330-008-1188-2

12. Takahashi S, Tanaka N, Okimoto T, et al. Long term follow-up for small pure ground-glass nodules: implications of determining an optimum follow-up period and high-resolution CT findings to predict the growth of nodules. Jpn J Radiol. 2012;30:206-217. doi:10.1007/ s11604-011-0033-8

13. Fan L, Liu SY, Li QC, Yu H, Xiao XS. Multidetector CT features of pulmonary focal ground-glass opacity: differences between benign and malignant. $B r J$ Radiol. 2012;85:897-904. doi:10.1259/bjr/ 33150223

14. Felix L, Serra-Tosio G, Lantuejoul S, et al. CT characteristics of resolving ground-glass opacities in a lung cancer screening programme. Eur $J$ Radiol. 2011;77:410-416. doi:10.1016/j. ejrad.2009.09.008

15. Li F, Sone S, Abe H, Macmahon H, Doi K. Malignant versus benign nodules at CT screening for lung cancer: comparison of thin-section CT findings. Radiology. 2004;233:793-798. doi:10.1148/ radiol.2333031018 
16. Nambu A, Araki T, Taguchi Y, et al. Focal area of ground-glass opacity and ground-glass opacity predominance on thin-section CT: discrimination between neoplastic and non-neoplastic lesions. Clin Radiol. 2005;60:1006-1017. doi:10.1016/j.crad.2005.06.006

17. Yang W, Sun Y, Fang W, et al. High-resolution computed tomography features distinguishing benign and malignant lesions manifesting as persistent solitary subsolid nodules. Clin Lung Cancer. 2018;19:e75e83. doi:10.1016/j.cllc.2017.05.023

18. Oh JY, Kwon SY, Yoon HI, et al. Clinical significance of a solitary ground-glass opacity (GGO) lesion of the lung detected by chest CT. Lung Cancer. 2007;55:67-73. doi:10.1016/j.lungcan.2006.09.009

19. Lee SM, Park CM, Goo JM, et al. Transient part-solid nodules detected at screening thin-section CT for lung cancer: comparison with persistent part-solid nodules. Radiology. 2010;255:242-251. doi:10.1148/radiol.09090547

20. Zhao F, Yan SX, Wang GF. CT features of focal organizing pneumonia: an analysis of consecutive histopathologically confirmed 45 cases. Eur J Radiol. 2014;83(1):73-78. doi:10.1016/j. ejrad.2013.04.017

21. de Hoop B, Gietema H, van de Vorst S, Murphy K, van Klaveren RJ, Prokop M. Pulmonary ground-glass nodules: increase in mass as an early indicator of growth. Radiology. 2010;255:199-206. doi:10.1148/radiol.09090571

22. Gao F, Li M, Ge X, et al. Multi-detector spiral CT study of the relationships between pulmonary ground-glass nodules and blood vessels. Eur Radiol. 2013;23:3271-3277. doi:10.1007/s00330-0132954-3
23. Lv YG, Bao JH, Xu DU, et al. Characteristic analysis of pulmonary ground-glass lesions with the help of 64-slice CT technology. Eur Rev Med Pharmacol Sci. 2017;21:3212-3217.

24. Naidich DP, Bankier AA, MacMahon H, et al. Recommendations for the management of subsolid pulmonary nodules detected at CT: a statement from the Fleischner Society. Radiology. 2013;266:304-317. doi:10.1148/radiol.12120628

25. Baldwin DR, Callister ME; Guideline Development Group. The British Thoracic Society guidelines on the investigation and management of pulmonary nodules. Thorax. 2015;70:794-798. doi:10.1136/ thoraxjnl-2015-207221

26. Hiramatsu M, Inagaki T, Inagaki $T$, et al. Pulmonary ground-glass opacity (GGO) lesions-large size and a history of lung cancer are risk factors for growth. $J$ Thorac Oncol. 2008;3:1245-1250. doi:10.1097/JTO.0b013e318189f526

27. Libby DM, Wu N, Lee IJ, et al. CT screening for lung cancer: the value of short-term CT follow-up. Chest. 2006;129:1039-1042. doi:10.1378/chest.129.4.1039
International Journal of General Medicine

\section{Publish your work in this journal}

The International Journal of General Medicine is an international, peer-reviewed open-access journal that focuses on general and internal medicine, pathogenesis, epidemiology, diagnosis, monitoring and treatment protocols. The journal is characterized by the rapid reporting of reviews, original research and clinical studies

\section{Dovepress}

across all disease areas. The manuscript management system is completely online and includes a very quick and fair peer-review system, which is all easy to use. Visit http://www.dovepress.com/ testimonials.php to read real quotes from published authors. 